\title{
Perturbative evolution of nonlinear initial data for binary black holes: Zerilli versus Teukolsky equation
}

\author{
Carlos O. Lousto \\ Albert-Einstein-Institut, Max-Planck-Institut für Gravitationsphysik, Am Mühlenberg 1, D-14476 Golm, Germany \\ and Instituto de Astronomía y Física del Espacio-CONICET, Buenos Aires, Argentina
}

(Received 17 June 1999; revised manuscript received 7 October 1999; published 30 January 2001)

\begin{abstract}
We consider the problem of evolving nonlinear initial data in the close limit regime. For exact Misner initial data (two equal mass black holes initially at rest), metric perturbations evolved via the Zerilli equation suffer from a premature breakdown (at a proper separation of the holes $L / M \approx 2.2$ ) while we find that the exact Weyl scalar $\psi_{4}$ evolved via the Teukolsky equation keeps very good agreement with the full numerical results up to $L / M \approx 3.5$. Metric and curvature perturbations of nonrotating black holes are equivalent to first perturbative order, but the Moncrief waveform in the former case and the Weyl scalar $\psi_{4}$ in the latter differ when nonlinearities are present. We argue that this inequivalent behavior holds for a wider class of conformally flat initial data.
\end{abstract}

DOI: 10.1103/PhysRevD.63.047504

PACS number(s): 04.30.Db, 04.25.Nx, 04.70.-s

There has been a revival of interest in the perturbation theory of black holes since the work of Price and Pullin [1] who put forward the close limit approximation. This approach considers the final merger stage of binary black holes as a single, perturbed black hole. They studied the Misner problem, two equal mass black holes initially at rest, and compared their results with full numerical evolution of the Einstein equations for various initial separations. The impressive agreement, even for not so small separations, triggered several researchers to test these ideas for initial data representing boosted, single spinning plus Brill waves and orbiting black holes [2].

Misner [3] found a solution to the conformally flat, time symmetric initial value problem representing two black holes at rest separated by a proper distance $L$ parametrized by $\mu_{0}$ (see below). For $\mu_{0}<1.8(L / M<3.3)$ a common event horizon encompasses the system, and for $\mu_{0}<1.36(L / M$ $<2.5)$ a common apparent horizon appears. The Misner three-geometry takes a conformal factor $\Phi(r, \theta)^{4}$ where

$$
\begin{aligned}
\Phi= & \left\{1+\delta \sum_{n \neq 0}\left[\left(1+\delta^{2}\right) \sinh ^{2} n \mu_{0}\right.\right. \\
& \left.\left.+\delta \cos \theta \sinh 2 n \mu_{0}+\delta^{2}\right]^{-1 / 2}\right\} /\left(1+\frac{M}{2 R}\right),
\end{aligned}
$$

where $\delta=M /\left(4 R \Sigma_{1}\right)$, and $\Sigma_{1} \dot{=} \Sigma_{n=1}^{\infty} 1 / \sinh n \mu_{0}$. Here we identified $R$, the conformal space radial coordinate, with the Schwarzschild isotropic coordinate, $R \doteq \frac{1}{4}(\sqrt{r}+\sqrt{r-2 M})^{2}$.

This metric represents an asymptotically flat threegeometry with total Arnowitt-Deser-Misner (ADM) mass $M$. The proper distance $L$ between the throats can be written as

$$
L=\frac{M}{2 \Sigma_{1}}\left(1+2 \mu_{0} \sum_{n=1}^{\infty} \frac{n}{\sinh n \mu_{0}}\right) .
$$

Abrahams and Price [4] found that if one does not linearize Misner initial data, but extracts the $l$ multipoles from the exact three-metric, the close limit approximation breaks down for much smaller separations, in a regime where the linearized approach precisely agrees with full numerical calculations. To linearize the three-metric (1) one can use the perturbative notion of $\Phi^{4}-1 \ll 1$. In this case, after expansion into Legendre polynomials, one gets

$$
\Phi^{4} \approx 1+\frac{8}{\left(1+\frac{M}{2 R}\right)} \sum_{l=2,4, \ldots} \kappa_{l}\left(\mu_{0}\right)(M / R)^{l+1} P_{l}(\cos \theta),
$$

where $\kappa_{l}\left(\mu_{0}\right) \doteq 1 /\left(4 \Sigma_{1}\right)^{l+1} \Sigma_{n=1}^{\infty}\left(\operatorname{coth} n \mu_{0}\right)^{l} / \sinh n \mu_{0}$. Note that the expansion parameter, $M \operatorname{coth}\left(\mu_{0}\right) /\left(4 R \Sigma_{1}\right)$, has to be $<1$ for the above expansion to be valid. This condition is always satisfied outside the effective single hole event horizon, located at $R=M / 2$, for $\mu_{0}<1.56865$. For larger values of $\mu_{0}$ one has an inverse expansion analogous to that made in Eq. (2.21) of Ref. [5].

Since to first order we only have quadrupolar $(l=2)$ contributions, the expansion (3) has the nice feature of separating, in the common factor $\kappa_{2}$, all the dependence on the initial distance between holes. This allows us to compute the whole family of evolutions with only one integration of the Zerilli equation, and then rescale the waveforms by their corresponding $\kappa_{2}\left(\mu_{0}\right)$. In Regge-Wheeler notation one finds that the only nonvanishing components of the perturbed three-metric are $\mathrm{H}_{2}=K$. One then builds up the initial value of the Moncrief waveform [14], $Q^{l}$ (with $\partial_{t} Q^{l}=0$ ) and evolves to obtain waveforms, spectra, and radiated energies [1].

It was first found by Abrahams and Price [4] that if one does not linearize the initial data, but extracts its multipoles from the exact metric as

$$
H_{2}^{(l 0)}=K^{(l 0)}=\int d \theta \sin \theta \Phi^{4} Y_{l 0}
$$




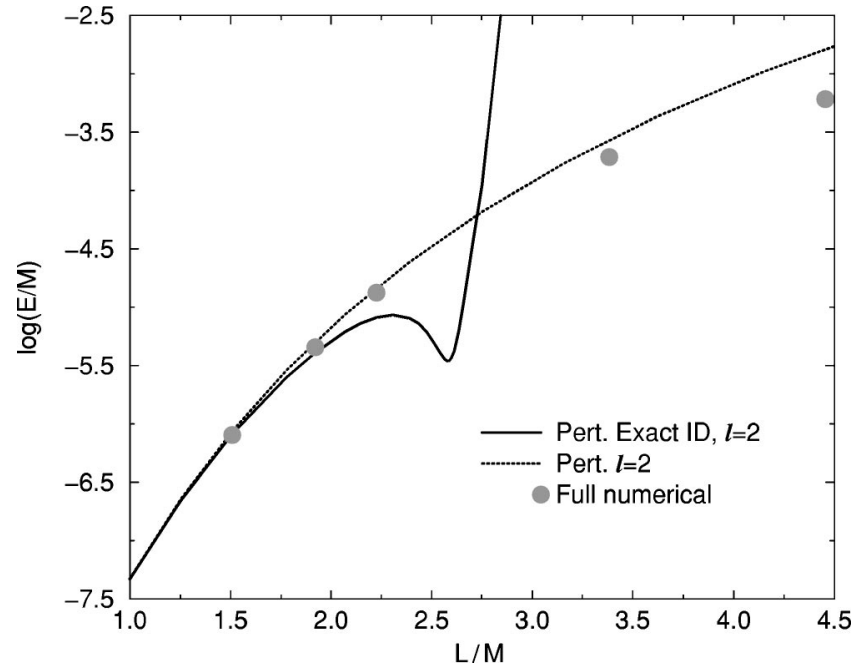

FIG. 1. The solid curve represents the total radiated energy $(E)$ computed via the Zerilli equation for two equal mass holes. At the bottom of the $\operatorname{dip}(L \approx 2.58)$ the system radiates one order of magnitude less energy than that predicted by the evolution of linearized initial data (dotted line).

and evolves using the Zerilli equation, one obtains for the radiated energy the disturbing results reproduced in Fig. 1. While for small separations $L / M<2$, corresponding to $\mu_{0}$ $<1.0$, the agreement of full numerical computations [6] and linearized perturbations is good; the energy curve presents a local maximum at $\mu_{0} \approx 1.25$ and then decreases to a local minimum at $\mu_{0} \approx 1.41$. At this point the system radiates an order of magnitude less than the evolution of linearized initial data. For larger values of $\mu_{0}$ the radiated energy rapidly increases, and soon after $\mu_{0} \approx 1.49$, it overestimates the total energy radiated by several orders of magnitude. All this happens well before the linearized theory begins to deviate from the full numerical results $\left(\mu_{0}>1.8\right)$. To unravel this paradoxical result we will first compute the corresponding radiated energies in an alternative formulation of the black hole perturbations.

There is an independent formulation of the perturbation problem derived from the Newman-Penrose formalism [7] that fully exploits the null structure of black holes allowing one to uncouple Einstein equations (and Bianchi identities) for a single wave equation to describe perturbations around Kerr black holes. The two approaches are related and equivalent when one deals with first order perturbations $[8,9]$. Here we want to explore how they behave under nonlinear components present in the initial data. The outgoing gravitational radiation is fully described in this gauge (and tetrad) invariant formalism by the Weyl scalar $\psi_{4}=-C_{\alpha \beta \gamma \delta} n^{\alpha} \bar{m}^{\beta} n^{\gamma} \bar{m}^{-}$, where $n^{\mu}$ and $\bar{m}^{\mu}$ (together with $l^{\mu}$ and $m^{\mu}$ ) form the tetrad that spans the spacetime.

The first step towards building up the initial $\psi_{4}$ and $\partial_{t} \psi_{4}$ is to find an instantaneous exact tetrad compatible with the data (1). We have found it by choosing the $l^{\mu}$ and $n^{\mu}$ that generate shear free null congruences (spin coefficients $\sigma$ $=0=\lambda)$ and fix the form of $m^{\mu}$ and its complex conjugate $\bar{m}^{\mu}$ under transformations of type III (boosts) in such a way

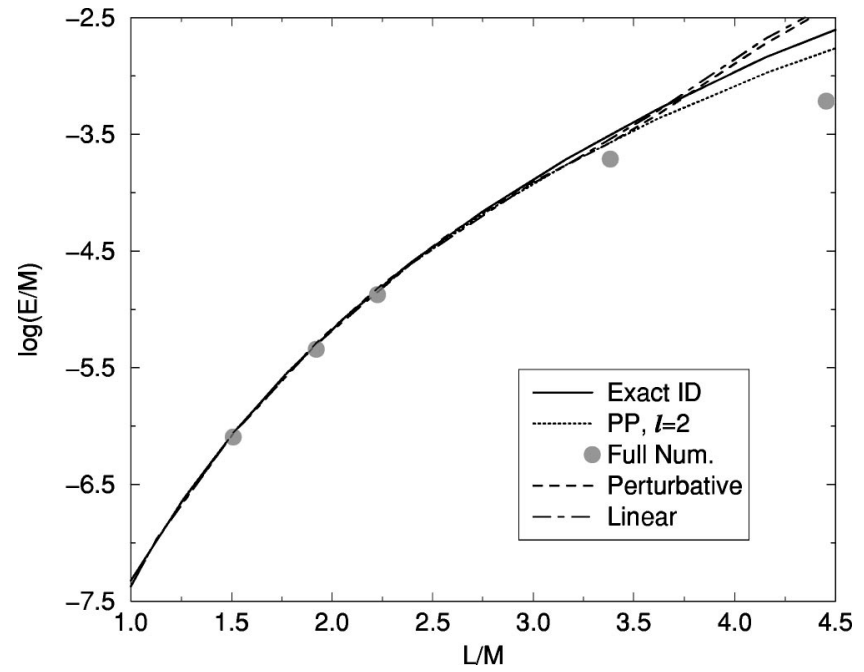

FIG. 2. The solid line gives the total radiated energy $(E)$ as computed using the Newman-Penrose-Teukolsky approach. There is complete agreement with full numerical results for $L<3.3$ and with the Price-Pullin results (labeled as PP, $l=2$ ) all the way up to much larger initial separations. Also shown are two other notions of perturbative initial data in the Newman-Penrose formalism.

that the spin coefficient $\epsilon=0$. Our tetrad is then

$$
\begin{aligned}
\left(l^{\mu}\right) & =\left(\frac{1}{1-2 M / r}, \Phi^{-2}, 0,0\right), \\
\left(n^{\mu}\right) & =\frac{1}{2}\left(1,-(1-2 M / r) \Phi^{-2}, 0,0\right), \\
\left(m^{\mu}\right) & =\frac{\Phi^{-2}}{\sqrt{2} r}(0,0,1, i / \sin \theta) .
\end{aligned}
$$

Using formulas (3.1) and (3.2) of Ref. [10], which give $\psi_{4}$ and $\partial_{t} \psi_{4}$ in terms of the three-geometry and the extrinsic curvature (here vanishing), we obtain

$$
\begin{aligned}
\left.\psi_{4}\right|_{t=0} & =\frac{(1-2 M / r)}{2 r^{2} \Phi^{6}}\left\{-3\left(\partial_{\theta} \Phi\right)^{2}+\Phi\left(\partial_{\theta}^{2}-\cot \theta \partial_{\theta}\right) \Phi\right\}, \\
\left.\partial_{t} \psi_{4}\right|_{t=0} & =-\left.\frac{2 M}{r^{2} \Phi^{2}} \psi_{4}\right|_{t=0} .
\end{aligned}
$$

The evolution of these initial data via the Teukolsky equation produces the results shown in Fig. 2 for the total energy emitted as gravitational waves. There is no dip at any value of the separation of the holes. The predicted energy agrees with the full numerical results for all $\mu_{0}<1.8$. For larger values of the separation (although we evolved here exact initial data for two distinct black holes) we overestimate the radiated energy at practically the same rate as the original result of Price and Pullin who extrapolated the linearized, $l$ $=2$, initial data to values of $\left(\mu_{0}>1.58\right)$ beyond the radius of convergence of their expansion parameter, i.e., $M \operatorname{coth}\left(\mu_{0}\right) /\left(4 R \Sigma_{1}\right)>1$. Correcting for this fact produces the $l=2$ piece of the curve labeled as "Linear." 


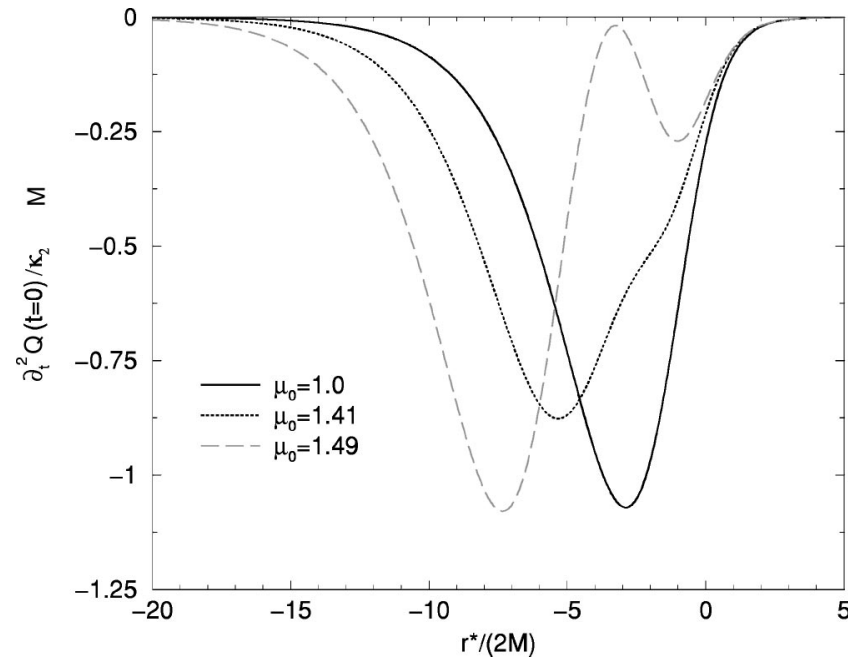

FIG. 3. The initial second time derivative of the Moncrief waveform normalized by $\kappa_{2}$ to compare with the linear initial data. While there is a good superposition of the data for $r^{*}>0$, the location of the maximum amplitude monotonically recedes towards more negative $r^{*}$ as $\mu_{0}$ increases. This provokes a decrease of the frequency for which destructive interference of the outgoing radiation occurs.

We also essayed two possible definitions of the perturbative notion in the Newman-Penrose formalism. The first one, which we called perturbative, considers the exact Weyl tensor contracted with the background (Schwarzschild) tetrad and a second possibility, which we called linear, considers only linear terms in the conformal factor $\Phi$. The resulting energy is essentially unchanged in the $\mu_{0}<1.8$ regime and grows steeper than the exact initial data for larger initial separations of the holes. This is because for the initial perturbative $\psi_{4}$ 's higher $l$ contributions are less under control than for the exact initial $\psi_{4}$. This is seen in the waveforms extracted far away from the system. While for the perturbative choices of $\psi_{4}$ waveforms look fine up to $\mu \approx 1.6$ those evolved from the exact $\psi_{4}$ reach $\mu \approx 2.0$ without much higher $l$ content. No dip was found at any value of the separation of the holes and in fact we could not reproduce the results of Fig. 1 within the Newman-Penrose-Teukolsky formalism. This gives us a measure of a certain robustness of this approach against nonlinearities included in the initial data.

Since both evolution equations, Zerilli's and Teukolsky's, are equivalent in the linear perturbations regime, the different results obtained must be related to how $\psi_{4}$ and $Q$ handle the nonlinearities included in the Misner data. The quantity directly related to the radiation $\partial_{t} Q$ initially vanishes for Misner data (time symmetric); in Fig. 3, we plot $\partial_{t}^{2} Q$ at $t$ $=0$. We observe a good superposition for $r^{*}>0$. There is also a big depression for linear data with a minimum at $r^{*} / 2 M \approx-3$. As the two black holes start from larger initial separations ( $\mu_{0}$ increases), the amplitude of this depression decreases and its location recedes towards more negative $r^{*}$ 's. This continues thus until we reach $\mu_{0} \approx 1.41$. Then the amplitude begins to grow while still the location of the depression recedes towards negative $r^{*}$, s. Is this relatively

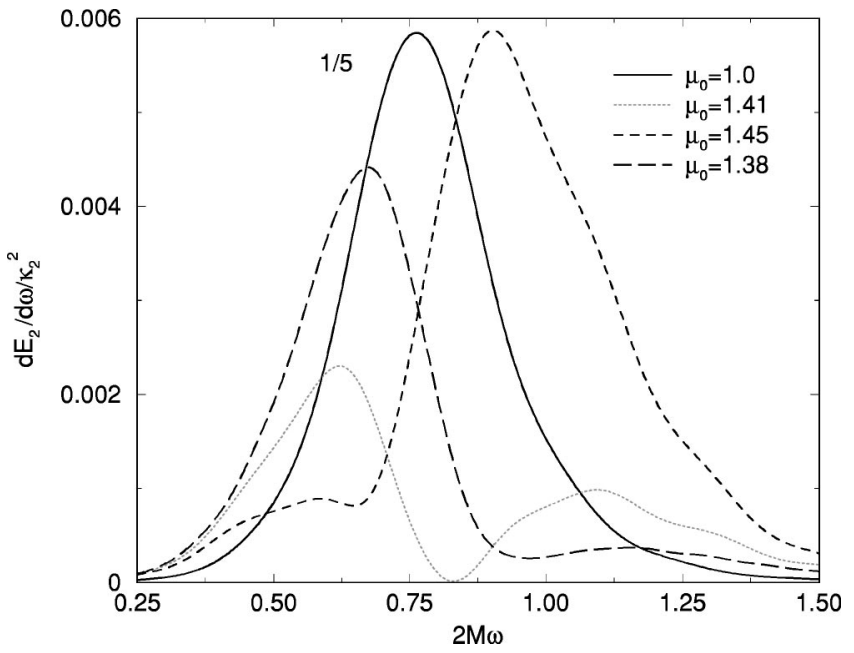

FIG. 4. The spectrum of the gravitational radiation as evolved by the Zerilli equation. The solid line labeled with the separation parameter $\mu_{0}=1$ gives essentially the linear regime (we rescaled its amplitude by one-fifth). We see how the suppression effect sets up sharply around $\mu_{0}=1.41$. For $\mu_{0}=1.38,1.41,1.45$ destructive interference occurs around frequencies $2 M \omega \approx 0.95,0.83,0.65$, respectively.

small decrease of the amplitude (around 20\%) responsible for one order of magnitude less radiated energy? To answer the above question one has to take into account the wave nature of the gravitational radiation. We know that a great deal of the radiation reaching infinity is generated around the maximum of the Zerilli potential at $r_{\text {max }}^{*} / 2 M \approx 0.95$. There will also be a piece of the disturbance generated at around the pick of $\partial_{t}^{2} Q$. These two pulses will be out of phase by $(2 M \omega)(\Delta t / 2 M)$ where $\Delta t$ is the time the pulse generated close to the horizon takes to arrive at $r^{*} / 2 M \approx 0.95$. Depending on their relative phases these two pulses can produce constructive or destructive interference. Assuming $\Delta t$ $\sim \Delta r^{*}$, where $\Delta r^{*}$ is the distance between the pick of $\partial_{t}^{2} Q$ and the maximum of the Zerilli potential, this analysis shows, that in the linear regime the destructive interference appears for frequencies $2 M \omega>1.2$, too high to influence the total energy radiated at infinity. As we increase $\mu_{0}$, the pick in Fig. 3 moves to the left, thus generating destructive interference at lower frequencies. In Fig. 4 we show that this effect is clearly visible in the three spectra for $\mu_{0}=1.38$, $1.41,1.45$. There is destructive interference around $2 M \omega$ $\approx 0.95,0.83,0.65$, respectively. The strong suppression of the radiation at $\mu_{0}=1.41$ is then due to destructive interference right at the frequency of the maximum of the linear spectrum, at $2 M \omega \sim 0.8$. By the same mechanism we can explain the sudden increase in the radiated energy for $\mu_{0}$ $>1.4$. It is now the effect of destructive interference acting at too low frequencies and constructive interference at higher ones together with a dramatic increase of the amplitude of the initial data with respect to the linear regime. Finally for $\mu_{0}>1.56865$ the effect of the change in the perturbative parameter makes things blow up.

There remains the question of why nothing like this happens when we use the Newman-Penrose-Teukolsky approach 
to Schwarzschild perturbations. Again, a plot of the initial data for different separations leads to the answer. We observe that the maximum of the initial data for increasing $\mu_{0}$ shifts toward increasing $r^{*}$ 's instead of more negative ones as happened for the Zerilli formalism. Thus regardless of a small decrease in the amplitude the destructive interference occurs at too high frequencies to influence the outgoing radiation.

Our results are relevant to the idea of evolving fully numerically binary black holes starting from large separations (starting from post-Newtonian initial data) until a common horizon encompasses the system and then let perturbation theory to take over [11]. The perturbation taking over the full numerical method has the advantage of optimizing supercomputer resources, concentrating them in the region where the two black holes are completely detached and full nonlinear relativistic effects take place. Once a common horizon forms one can assume the close limit approximation to hold and continue the evolution with a single wave equation on the background of a Kerr black hole. It is very fortunate that is the Newman-Penrose-Teukolsky approach rather than the Regge-Wheeler-Zerilli one that has this nice behavior in response to nonlinear Cauchy data since the Teukolsky equation can be generalized to rotating black hole backgrounds while the metric perturbation approach cannot. The effect described in Fig. 1 inhibits us from using the Zerilli equation to that end. On the other hand, the Teukolsky evolution seems better suited for this marriage between full numerical and perturbative approaches. We also checked that the same general bad behavior of the Zerilli equation and healthy one for the Teukolsky equation hold when one considers Brill-
Lindquist initial data (other solutions of the conformally flat, time symmetric initial value problem). This behavior is also true for orbiting black holes if we remain within the BowenYork family of initial data (conformally flat and longitudinal). In this case we can make a decomposition of the conformal factor of the type [12] $\Phi=\Phi_{\text {Misner }}+\Phi_{\text {reg }}$, where $\Phi_{\text {reg }}$ is proportional to the square of the momentum of the holes and the square of their distance. This means that at least for orbiting black holes with small initial momentum the effects discussed in this paper should qualitatively still be present.

Another situation where our results should be considered is when one is interested in studying second order perturbations of rotating black holes [13]. The perturbative approach to the Newman-Penrose formalism forms a hierarchy of equations

$$
\hat{\mathcal{T}} \psi_{4}^{(N)}=\mathcal{S}\left[\psi^{(N-1)}, \partial_{t} \psi^{(N-1)}\right]
$$

where $\mathcal{T}$ stands for the background wave operator of the Teukolsky equation, $\psi_{4}^{(N)}$ is the waveform of the $N$ perturbative order considered, and $\mathcal{S}$ is a source term formed by products of all perturbations of order lower than $N$. One can solve the above equations by evolving the initial data order by order, successively reaching the next perturbative stage. An alternative approach to that is to evolve exact initial data with the first order wave equation (it has a vanishing source term in vacuum), and then evolve second order equations with vanishing initial data (expressing the source in terms of the first order perturbations). This is consistent with the desired second perturbative order.
[1] R. Price and J. Pullin, Phys. Rev. Lett. 72, 3297 (1994).

[2] J. Pullin, in Proceedings of the GR15, edited by N. Dadhich and J. Narlikar (Inter-University Centre for Astronomy and Astrophysics, Puna, 1998), p. 87.

[3] C. W. Misner, Ann. Phys. (N.Y.) 24, 102 (1963); R. W. Lindquist, J. Math. Phys. 4, 938 (1963).

[4] A. Abrahams and R. Price, Phys. Rev. D 53, 1963 (1996).

[5] C. O. Lousto and R. Price, Phys. Rev. D 55, 2124 (1997).

[6] P. Anninos, D. Hobil, E. Seidel, L. Smarr, and W.-M. Suen, Phys. Rev. D 52, 2044 (1995).

[7] S. A. Teukolsky, Astrophys. J. 185, 635 (1973).

[8] M. Campanelli and C. O. Lousto, Phys. Rev. D 58, 024015
(1998).

[9] M. Campanelli, W. Krivan, and C. O. Lousto, Phys. Rev. D 58, 024016 (1998).

[10] M. Campanelli, C. O. Lousto, J. Baker, G. Khanna, and J. Pullin, Phys. Rev. D 58, 084019 (1998).

[11] J. Baker, B. Brügmann, M. Campanelli, and C. Lousto, Class. Quantum Grav. 17, L149 (2000).

[12] C. O. Nicasio, R. J. Gleiser, R. H. Price, and J. Pullin, Phys. Rev. D 59, 044024 (1999).

[13] M. Campanelli and C. O. Lousto, Phys. Rev. D 59, 124022 (1999).

[14] V. Moncrief, Ann. Phys. (N.Y.) 88, 323 (1974). . 